\title{
Pregnancy outcome in a bicornuate uterus with bilateral pregnancy presenting as threatened abortion: a case report
}

\author{
Tanaya Acharyya ${ }^{1 *}$, Bandana Shyam Gohain ${ }^{1}$, Kripanath Morang²
}

\author{
${ }^{1}$ Department of Obstetrics and Gynaecology, Silchar Medical College and Hospital, Assam, India \\ ${ }^{2}$ Department of Radiology, Silchar Medical College and Hospital, Assam, India
}

Received: 05 September 2021

Accepted: 01 October 2021

\author{
*Correspondence: \\ Dr. Tanaya Acharyya, \\ E-mail: Tanayaacharyya.tiarra@gmail.com
}

Copyright: $@$ the author(s), publisher and licensee Medip Academy. This is an open-access article distributed under the terms of the Creative Commons Attribution Non-Commercial License, which permits unrestricted non-commercial use, distribution, and reproduction in any medium, provided the original work is properly cited.

\section{ABSTRACT}

Congenital uterine anomalies or mullerian anomalies are prevalent in 0.4 to $10 \%$ of women in general and are often manifested by reproductive challenges like miscarriage, premature labor, premature rupture of membranes or malpresentation. Having a bilateral pregnancy in a bicornuate uterus is extremely rare, especially if it is a spontaneous conception.

Keywords: Bicornuate uterus, Congenital uterine malformation, Threatened abortion

\section{INTRODUCTION}

Congenital uterine anomalies or mullerian anomalies result from abnormal formation, fusion or resorption of mullerian duct during fetal life. One of these abnormalities is identified as bicornuate uterus which is caused by abnormal fusion of mullerian ducts. Prevalence found in general population is approximately 0.4 to $10 \%$. According to ESHRE classification, bicorporeal uterus is class U3, can be partial, complete and bicorporeal septate. ${ }^{1}$

The prevalence of mullerian anomalies in association with pregnancy is not so uncommon, but it is difficult to assess antenatally because best diagnostic techniques are invasive. Many pregnancies with bicornuate uterus remain asymptomatic but should be suspected in patients with recurrent miscarriages and malpresentations. However, women with bicornuate uterus can experience successful pregnancies and even uneventful vaginal deliveries.

One such case of successful pregnancy outcome in a patient with complete bicornuate uterus with bilateral pregnancy with a history of spontaneous abortion in one horn has been discussed in this case report. The final pregnancy outcome in the other horn was indeed exemplary with appropriate treatment and proper follow up.

\section{CASE REPORT}

A 28 years old woman, second gravida with one living issue with history of once prior cesarean section came to our antenatal OPD of SMCH with complains of pain abdomen and bleeding per vaginum in her 8th week of gestation. She couldn't specify any history of uterine abnormality reported in her previous pregnancy. According to her, indication of previous CS was prolonged labor. Transabdominal sonography done on the day of her visit to our OPD, came out to be as bicorporeal uterus with bilateral pregnancy. At one horn a G-sac corresponding to 8 weeks 3 days \pm 6 days with definite fetal pole and cardiac activity was present. In the other horn, a deformed sac corresponding to 8 weeks 5 days \pm 6 days with no definite fetal pole and cardiac activity was seen. Patient was admitted as a case of threatened abortion and bed rest was advised along with weekly inj. HCG. The next day she had expelled the product of conceptus along with gush of bleeding. On repeat TAS, empty horn was noted on one 
side along with blood collection in vaginal canal and closed internal os suggesting complete abortion. Intact Gsac was noted in the other side. Blood clots were cleared off the introitus.

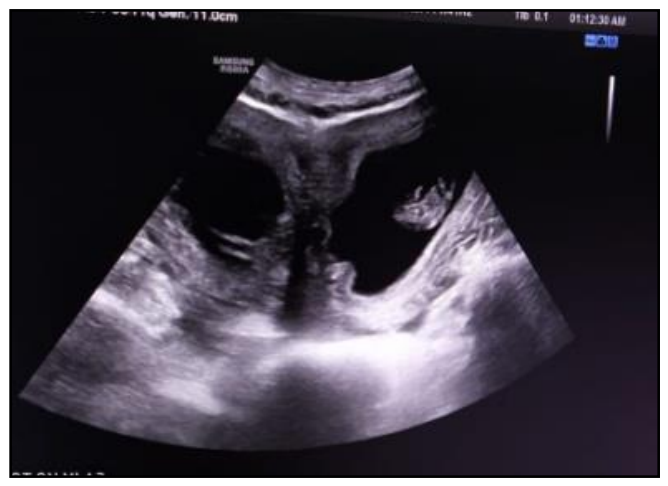

Figure 1: TAS Showing bicornuate uterus with deformed G. sac in one horn and live gestation in the other horn.

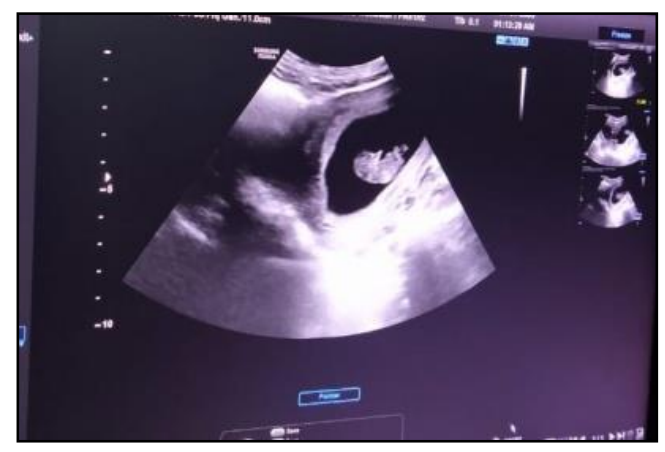

Figure 2: TAS showing bicornuate uterus with empty uterine cavity in one horn suggesting complete abortion and live gestation in the other horn.

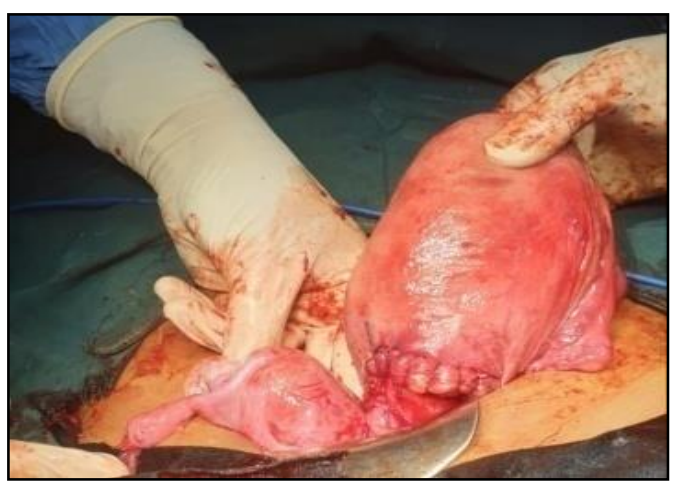

Figure 3: Intraoperative picture taken during Csection of the patient, showing bicornuate uterus.

Patient was managed conservatively and discharged the next day with the advice of bed rest, tranexemic acid tablets, micronized progesterone tablets and once weely injection HCG for 6 weeks. She was adviced to attend OPD for follow up on monthly basis. She came for her ANC twice after that, one at 26 weeks and another at 35 weeks.
Ultrasound done at 35 weeks showed breech presentation with adequate liquor and fetal weight $2.2 \mathrm{~kg}$. Thereafter, at 37 weeks of gestation, she came to labor room with pain abdomen. On examination, she was in labor and so emergency LSCS under SA was done. Fetal weight was $2.6 \mathrm{~kg}$ with no gross fetal malformation. Placenta was lying anteriorly and liquor volume was adequate. Apgar score taken at 1 min and 5 mins were 8 and 9 respectively. Bilateral tubal ligation was done taking proper consent. Post operative period was uneventful. Both the mother and the baby were healthy at discharge.

\section{DISCUSSION}

The prevalence of congenital anomalies of uterus associated with pregnancy ranges between $0.1 \%$ to $3 \%$. $^{2}$ The commonest congenital uterine anomalies are bicornuate uterus and septate uterus, whereas uterus didelphys and unicornuate uterus being the least common among all malformations.

Congenital uterine malformations are often asymptomatic. Reproductive outcome and pregnancy rates are poor in women with uterine anomalies. Compared with women who possess normal uterus, uterine anomalies most commonly results in increased incidence of malpresentation, premature labor, abnormal presentation with dystocia thus increasing the necessity for CS. Other adverse pregnancy outcomes associated with bicornuate uterus are spontaneous rupture, because of thin wall and inability of malformed uterus to expand as a normal one and also spontaneous abortion. Hence, pregnancies in bicornuate uterus are considered as high risk pregnancies.

The incidence of spontaneous bilateral or twin pregnancy in bicornuate uterus is extremely rare and is estimated to be as 1 in 10,00,000 cases and this case belongs to this rare category. The incidence is higher in patients taking ovulation induction drugs. ${ }^{3}$

Early ultrasound is a contributing method for evaluation of the effects of abnormal uterus on pregnancy. 3D-TVS has sensitivity of $96.7 \%$ and specificity of $100 \%$, whereas 2DTVS has sensitivity of $50-75 \%$ and specificity of $95 \% .4 \mathrm{In}$ this case report, USG could not identify the uterine anomaly in first pregnancy, probably because of small size of uterine horn or improper imaging. However, in her second pregnancy she was accurately diagnosed with bicornuate uterus in early trimester. Correct diagnosis in early pregnancy raises the awareness of patients as well as of the obstetrician towards possible outcome of the condition, thus preventing complications.

\section{CONCLUSION}

Although adverse pregnancy outcomes like spontaneous abortion had been documented in this case of bicornuate uterus with bilateral pregnancy, with early diagnosis and proper management this pregnancy managed to reach term and delivered with good fetal outcome. 
Funding: No funding sources

Conflict of interest: None declared

Ethical approval: Not required

\section{REFERENCES}

1. Grimbizis GF, Saravelos SH. The Thessaloniki ESHRE/ESGE consensus on diagnosis of female genital anomalies. Human Reprod. 2016;31:2-7.

2. Ilyas M, Dar MS. Twin pregnancy in bicornuate uterus-one fetus in each horn. Journal of Fetal Medicine. 2018;5(1):59-61.
3. Enebe JT. Retained second twin secondary to an undiagnosed bicornuate uterus in a poorly supervised labour-a case report. Proceedings of Singapore Healthcare. 2021;30(2)162-5.

4. Borgohain D. Case report pregnancy in bicornuate uterus. Int J Reprod Contracept Obstet Gynaecol. 2018;7(1):346-8

Cite this article as: Acharyya T, Gohain BS, Morang K. Pregnancy outcome in a bicornuate uterus with bilateral pregnancy presenting as threatened abortion: a case report. Int $\mathbf{J}$ Reprod Contracept Obstet Gynecol 2021;10:4334-6. 